Экспериментальное исследование влияния единичного числа Рейнольдса на положение ламинарно-турбулентного перехода на крыле с дозвуковой передней кромкой при числе Маха 2

\author{
В. Л. Кочарин ${ }^{1}$, Н. В. Семёнов ${ }^{1}$, А. Д. Косинов ${ }^{1}$, А. А. Яцких ${ }^{1,2}$ \\ С. А. Шипуль ${ }^{1}$, Ю. Г. Ермолаев ${ }^{1}$ \\ ${ }^{1}$ Институт теоретической и прикладной механики им С. А. Христиановича СО РАН \\ Новосибирск, Россия \\ ${ }^{2}$ Новосибирский государственный университет \\ Новосибирск, Россия
}

Аннотация

Проведены экспериментальные исследования влияния единичного числа Рейнольдса на ламинарно-турбулентный переход в сверхзвуковом пограничном слое скользящего крыла с дозвуковой передней кромкой при числе Маха 2. Эксперименты выполнены на модели скользящего крыла с углом скольжения передней кромки $72^{\circ}$ и задней кромки $58^{\circ}$ с $3 \%$ профилем у основания. Полученные термоанемометрические данные показали, что ламинарно-турбулентный переход в сверхзвуковом пограничном слое скользящего крыла с дозвуковой передней кромкой наступает раньше ( $\approx 25 \div 30 \%)$, чем на модели со сверхзвуковой передней кромкой при одних и тех же параметрах набегающего потока. Показано, что изменение единичного числа Рейнольдса набегающего потока слабо влияет на ламинарно-турбулентный переход в пограничном слое скользящего крыла с дозвуковой передней кромкой.

Ключевые слова

эксперимент, сверхзвуковой поток, ламинарно-турбулентный переход, турбулентность, пограничный слой, скользящее крыло

Источник финансирования

Работа выполнена при поддержке Российского фонда фундаментальных исследований (проект № 19-0800772) с использованием оборудования ЦКП «Механика» (ИТПМ СО РАН)

Для цичтирования

Кочарин В. Л., Семёнов Н. В., Косинов А. Д., Ячиких А. А., Шипуль С. А., Ермолаев Ю. Г. Экспериментальное исследование влияния единичного числа Рейнольдса на положение ламинарно-турбулентного перехода на крыле с дозвуковой передней кромкой при числе Маха 2 // Сибирский физический журнал. 2021. Т. 16, № 1. C. 44-52. DOI 10.25205/2541-9447-2021-16-1-44-52

\title{
Experimental Study of the Influence of Unit Reynolds Number on the Laminar-Turbulent Transition on the Model Swept Wing with a Subsonic Leading Edge at $\mathrm{M}=2$
}

\author{
V. L. Kocharin ${ }^{1}$, N. V. Semionov ${ }^{1}$, A. D. Kosinov ${ }^{1}$, A. A. Yatskikh ${ }^{1,2}$ \\ S. A. Shipul ${ }^{1}$, Yu. G. Yermolaev ${ }^{1}$ \\ ${ }^{1}$ Khristianovich Institute of Theoretical and Applied Mechanics SB RAS \\ Novosibirsk, Russian Federation \\ ${ }^{2}$ Novosibirsk State University \\ Novosibirsk, Russian Federation
}

Abstract

Experimental studies of the influence of unit Reynolds number on the laminar-turbulent transition in a supersonic boundary layer of a swept wing with a subsonic leading edge at Mach number 2 are performed. The experiments were

() В.Л. Кочарин, Н. В. Семёнов, А. Д. Косинов, А. А. Яцких,

С. А. Шипуль, Ю.Г. Ермолаев, 2021 
performed on a model of a swept wing with a swept angle of the leading edge of 72 degrees and with a $3 \%$ profile with a variable chord length in span. The hot-wire measurements showed that a laminar-turbulent transition in a supersonic boundary layer of a swept wing with a subsonic leading edge occurs earlier $(\approx 25 \div 30 \%)$ than on a model with a supersonic leading edge with the same oncoming flow parameters. It is shown that a change unit Reynolds number insignificant influence the laminar-turbulent transition in the boundary layer of a swept wing with a subsonic leading edge.

Keywords

experiment, supersonic flow, laminar-turbulent transition, turbulence, boundary layer, swept wing

Funding

The work is supported by Russian Foundation for Basic Research (grant No. 19-08-00772). The study was conducted at the Joint Access Center "Mechanics" of ITAM SB RAS

For citation

Kocharin V. L., Semionov N. V., Kosinov A. D., Yatskikh A. A., Shipul S. A., Yermolaev Yu. G. Experimental Study of the Influence of Unit Reynolds Number on the Laminar-Turbulent Transition on the Model Swept Wing with a Subsonic Leading Edge at $\mathrm{M}=2$. Siberian Journal of Physics, 2021, vol. 16, no. 1, p. 45-53. (in Russ.) DOI 10.25205/2541-9447-2021-16-1-45-53

\section{Введение}

Исследование ламинарно-турбулентного перехода на скользящем крыле при сверхзвуковых скоростях потока имеет практический интерес для развития высокоскоростной авиационной техники. Отметим, что имеется только несколько работ, в которых на трехмерных моделях крыла при сверхзвуковых скоростях потока проводились измерения положения ламинарно-турбулентного перехода. Обзор работ, где приводятся экспериментальные данные по влиянию единичного числа Рейнольдса на положение перехода на скользящем крыле в высокоскоростном потоке приведен в [1]. Обтекание скользящего крыла сверхзвуковым потоком принято классифицировать по числу Маха по нормали к передней кромке. Передняя кромка называется дозвуковой, если число Маха по нормали $\mathrm{M}^{\prime}<1$; звуковой, если $\mathrm{M}^{\prime}=1$, и сверхзвуковой, если $\mathrm{M}^{\prime}>1$. Отметим работы, где приводятся данные для случая дозвуковой передней кромки. Эксперименты на дельта-крыле с углом скольжения $74^{\circ}$ выполнены в баллистических трассах в диапазоне чисел Маха от 2,8 до 5,3 [2] для случаев дозвуковой и сверхзвуковой передней кромки (в зависимости от числа Маха) $(\mathrm{M}=2,81 ; 3,02 ; 3,24 ; 3,35$; 4,$\left.1 ; 5,3 \rightarrow \mathrm{M}^{\prime}=0,77 ; 0,83 ; 0,89 ; 0,92 ; 1,13 ; 1,46\right)$. Влияния единичного числа Рейнольдса на положение ламинарно-турбулентного перехода не было зафиксировано. Получено, что для случая дозвуковой передней кромки $\operatorname{Re}_{\mathrm{tr}}=3 \times 10^{6}$ при $\mathrm{M}=2,78, \operatorname{Re}_{\mathrm{tr}}=3,3 \times 10^{6}$ при $\mathrm{M}=3,35$, для сверхзвуковой передней кромки $\operatorname{Re}_{\text {tr }}=4,8 \times 10^{6}$ при $\mathrm{M}=4,1$ и $\operatorname{Re}_{\mathrm{tr}} \approx 3 \div 4 \times 10^{6}$ при $\mathrm{M}=5,3$. В работе [3] приводятся результаты измерений на модели дельта-крыла с углом скольжения $\chi=77,1^{\circ}$ (дозвуковая передняя кромка) при $\mathrm{M}=3,5$. Отмечается, что переход наблюдался параллельно боковой кромке модели и единичное число Рейнольдса оказывает влияние на положение перехода.

Измерения положения перехода при $\mathrm{M}=2$ на модели крыла с дозвуковой передней кромкой (углы скольжения внутренней и внешней частей крыла составляли 66 и $61,2^{\circ}$ соответственно) были выполнены при помощи термопленочных датчиков и инфракрасной камеры в [4]. Показано, что единичное число Рейнольдса не влияет на положение перехода, и при нулевом угле атаки число Рейнольдса перехода составляло примерно $0,8 \times 10^{6}$. Результаты летных измерений $\mathrm{Re}_{\mathrm{tr}}$ на скользящем крыле при $\mathrm{M}=2$ приведены в работе [5]. Передняя кромка крыла имела те же два угла скольжения, что и в работе [4]. Числа Рейнольдса перехода в работе не приведены, а из представленных данных можно только сказать, что переход происходит вблизи передней кромки.

Результаты экспериментальных исследований по влиянию единичного числа Рейнольдса на положение ламинарно-турбулентного перехода в пограничном слое на скользящем крыле при сверхзвуковых скоростях потока приведены в [1]. В экспериментах использовались 3 \% симметричная модель скользящего крыла с чечевицеобразным профилем и углом скольже- 
ния кромок $45^{\circ}$, что соответствует случаю сверхзвуковой передней кромки. Положение перехода определялось с помощью термоанемометра. Получено, что при $\mathrm{M}=2$ и 2,5 увеличение единичного числа Рейнольдса $\left(\mathrm{Re}_{1}\right)$ приводит к затягиванию перехода. Показано, что увеличение числа Маха набегающего потока и уровня пульсаций в рабочей части аэродинамической трубы приводит к уменьшению влияния $\operatorname{Re}_{1}$ на положение перехода. При высоком уровне шума, вызванного ростом числа Маха или введением вихревых возмущений, влияние не фиксируется.

В работе [6] приведены данные о возникновении турбулентности на модели эллиптического конуса с углом полураствора $7^{\circ}$ при $\mathrm{M}=6,5$, полученные для нескольких значений единичного числа Рейнольдса. По представленным в работе результатам визуализации нами выполнены оценки положения перехода у боковых кромок модели. Получено, что увеличение значения $\mathrm{Re}_{1}$ от $3 \times 10^{6}$ до $6,8 \times 10^{6} \mathrm{M}^{-1}$ приводит к затягиванию положения ламинарнотурбулентного перехода от $\operatorname{Re}_{\text {пер }} \approx 0,7 \times 10^{6}$ до $\approx 1 \times 10^{6}$.

Следовательно, вопрос о влиянии единичного числа Рейнольдса на положение ламинарнотурбулентного перехода в пограничном слое на скользящем крыле с дозвуковой передней кромкой остается открытым.

\section{Постановка экспериментов}

Эксперименты выполнены в малотурбулентной сверхзвуковой аэродинамической трубе Т-325 ИТПМ СО РАН при числе Маха $\mathrm{M}=2$. Измерения проводились на модели скользящего крыла с 3 \% профилем с изменяемой длиной хорды по размаху (хорда у основания - 498 и 200 мм в конце крыла). Угол скольжения передней кромки составляет $72^{\circ}$. Схема модели представлена на рис. 1.

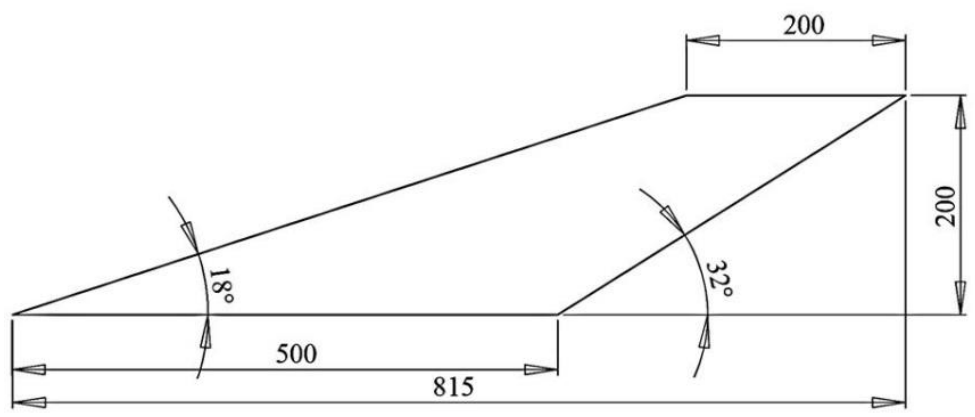

Puc. 1. Схема модели крыла с углом скольжения передней кромки $\chi=72^{\circ}$ Fig. 1. Scheme of a wing model with a sweep angle of the leading edge $\chi=72^{\circ}$

Возмущения в потоке регистрировались термоанемометром постоянного сопротивления. Датчики термоанемометра изготавливались из вольфрамовой нити диаметром 10 мкм и длиной около 1,5 мм. Величина перегрева нити датчика устанавливалась 0,8 , а измеренные возмущения преимущественно соответствовали пульсациям массового расхода. Измерения пульсационных и средних характеристик потока проводились автоматизированной системой сбора данных [7]. Обработка экспериментальных данных проводилась при помощи быстрого преобразования Фурье, определялись спектры мощности по полным осциллограммам. Более подробно постановка эксперимента, система сбора и процедура обработки данных описана в $[1 ; 7 ; 8]$.

В данных экспериментах использовались два способа определения положения ламинарнотурбулентного перехода с помощью термоанемометра: в первом случае измерения выполняются при фиксированном положении датчика, изменяется значение $\mathrm{Re}_{1}$, во втором - выбира- 
ется $\mathrm{Re}_{1}=$ const, а датчик перемещается вдоль координаты $x$. В результате измерений определялась зависимость амплитуды возмущений от числа Рейнольдса.

\section{Результаты}

Выполнено экспериментальное исследование влияния единичного числа Рейнольдса на положение ламинарно-турбулентного перехода в трехмерном сверхзвуковом пограничном слое на модели скользящего крыла с углом скольжения $72^{\circ}$, что соответствует случаю дозвуковой передней кромки при $\mathrm{M}=2$. На рис. 2 представлены зависимости интегральных среднеквадратичных пульсаций массового расхода от числа Рейнольдса $\operatorname{Re}_{x}=\operatorname{Re}_{1} \times x$, где $x-$ продольная координата, измеренных при фиксированных положениях датчика $x=80,100$, 119 мм при $\mathrm{M}=2,0$. Датчик устанавливался немного ниже области максимальных пульсаций поперек пограничного слоя при минимальных значениях $\mathrm{Re}_{1}$, тогда как при измерениях в переходной области датчик находился в окрестности максимума пульсаций. В результате измерений определялось значение числа Рейнольдса перехода, которое соответствовало максимуму кривой нарастания среднеквадратичных пульсаций. Из графика видно, что с увеличением единичного числа Рейнольдса положение перехода слабо смещается вниз по потоку.

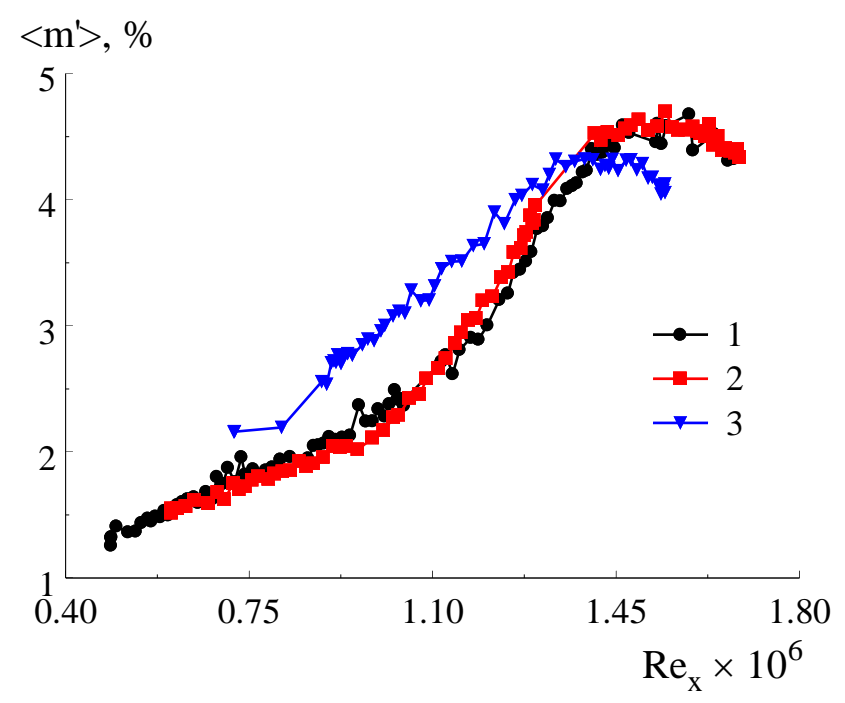

Puc. 2. Зависимость среднеквадратичных пульсаций массового расхода от числа Рейнольдса при $\mathrm{M}=2$.

Измерения по $\operatorname{Re}_{1}$ при $x=80$ мм (1), $x=100$ мм (2) и $x=119$ мм (3)

Fig. 2. Dependence of the rms pulsations on the Reynolds number at $\mathrm{M}=2$.

Measurements by $\operatorname{Re}_{1}$ at $x=80 \mathrm{~mm}(1), x=100 \mathrm{~mm}(2)$ and $x=119 \mathrm{~mm}(3)$

Незначительное влияние числа $\mathrm{Re}_{1}$ было зафиксировано при измерениях среднеквадратичных пульсаций массового расхода вниз по потоку при $\rho U \approx$ const для значений $\operatorname{Re}_{1}=14 \times 10^{6}$ и $18 \times 10^{6} \mathrm{M}^{-1}$ (рис. 3). В этом случае, при фиксированном значении единичного числа Рейнольдса, проводилось перемещение датчика по координате $x$ в области максимальных пульсаций по нормали к поверхности модели, а напряжение в диагонали моста термоанемометра поддерживалось постоянным за счет перемещения датчика по нормальной координате $y$. Получено, что увеличение единичного числа Рейнольдса $\operatorname{Re}_{1}$ приводит к росту числа Рейнольдса перехода $\mathrm{Re}_{\mathrm{tr}}$, как и в случае сверхзвуковой передней кромки [1]. По данным, представленным на рис. 2 и 3 были получены зависимости числа Рейнольдса перехода $\mathrm{Re}_{\text {tr }}$ от единичного числа Рейнольдса $\mathrm{Re}_{1}$ на модели скользящего крыла с дозвуковой передней кромкой $\left(\chi=72^{\circ}\right)$, представленные на рис. 4. Для сравнения на этом же графике 
представлены результаты измерений, выполненных на модели крыла с углом скольжения передней кромки $\chi=45^{\circ}$ из работы [1]. Получено, что ламинарно-турбулентный переход в сверхзвуковом пограничном слое скользящего крыла с дозвуковой передней кромкой наступает раньше $(\approx 25 \div 30 \%)$, чем на модели со сверхзвуковой передней кромкой при одних и тех же параметрах набегающего потока. Причиной более раннего перехода и слабого влияния $\operatorname{Re}_{1}$ может быть определяющая роль стационарных возмущений в процессе ламинарнотурбулентного перехода на модели скользящего крыла с дозвуковой передней кромкой.

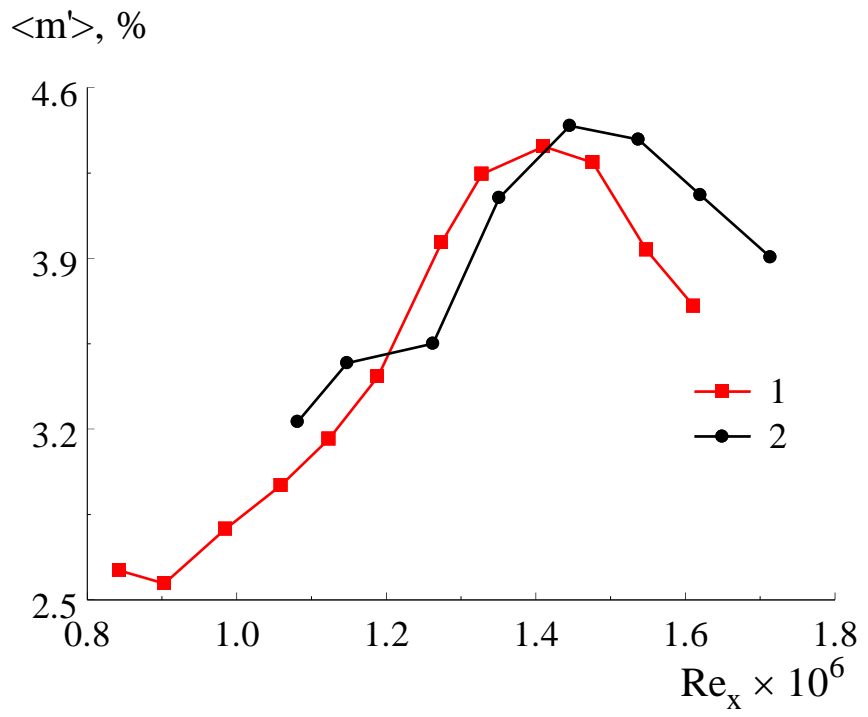

Puc. 3. Зависимость среднеквадратичных пульсаций массового расхода от числа Рейнольдса. Измерения по $x$ при $\operatorname{Re}_{1}=14 \times 10^{6} \mathrm{M}^{-1}(1)$ и $\mathrm{Re}_{1}=18 \times 10^{6} \mathrm{M}^{-1}(2)$

Fig. 3. Dependence of rms pulsations on the Reynolds number.

Measurements in $\mathrm{x}$ at $\mathrm{Re}_{1}=14 \times 10^{6} \mathrm{~m}^{-1}(1)$ and $\mathrm{Re}_{1}=18 \times 10^{6} \mathrm{~m}^{-1}(2)$

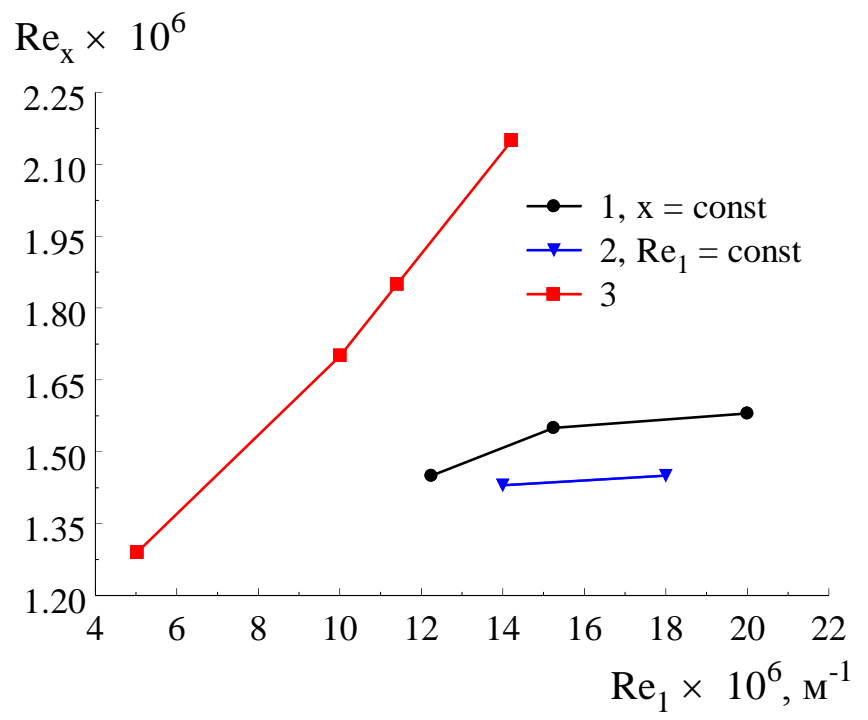

Puc. 4. Зависимости числа Рейнольдса перехода $\mathrm{Re}_{\mathrm{tr}}$ от единичного числа Рейнольдса $\mathrm{Re}_{1}$ на моделях со скользящим крылом с дозвуковой $\left(\chi=72^{\circ}\right)(1,2)$

и со сверхзвуковой передней кромкой $\left(\chi=45^{\circ}\right)(3)$ при $\mathrm{M}=2$

Fig. 4. Dependences of the Reynolds number of the transition $\operatorname{Re}_{\text {tr }}$ on the unit Reynolds number $\operatorname{Re}_{1}$ on models of swept wings with a subsonic leading edge $\left(\chi=72^{\circ}\right)(1,2)$ and with a supersonic leading edge $\left(\chi=45^{\circ}\right)(3)$ at $\mathrm{M}=2$ 


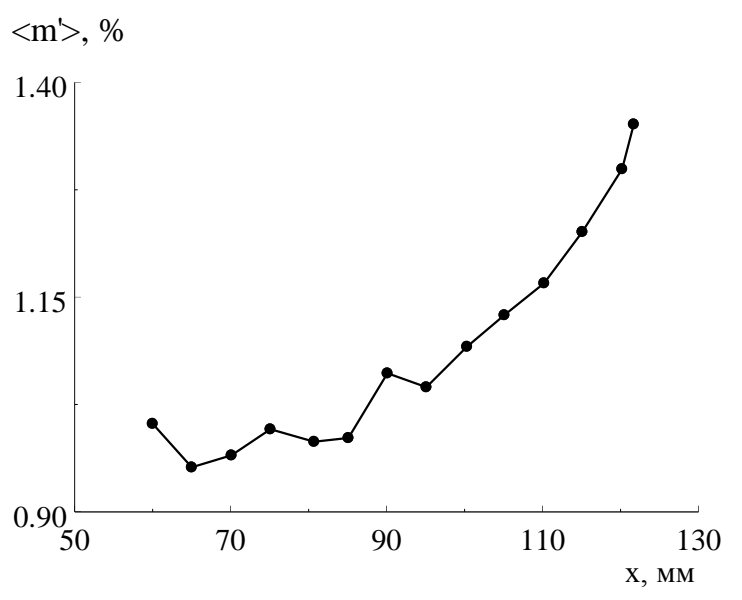

a

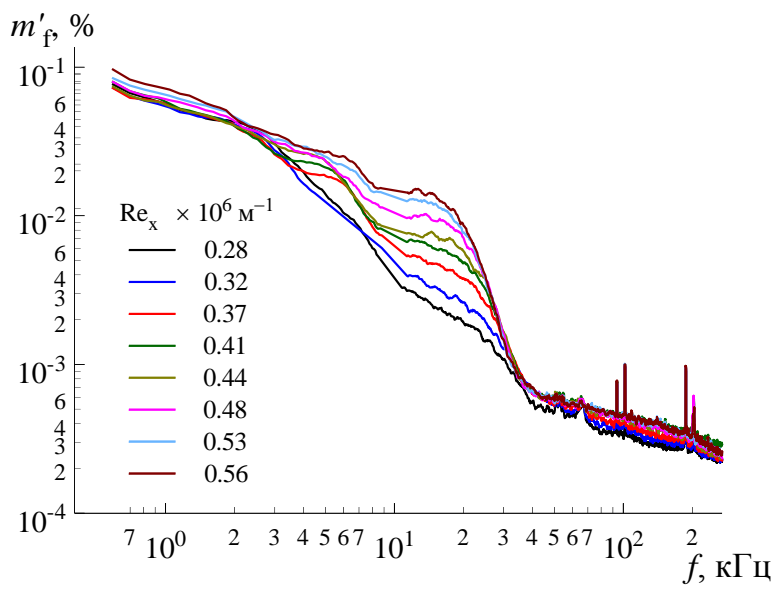

6

Puc. 5. Кривая нарастания возмущений (a) при $z=60$ мм и эволюция амплитудно-частотных спектров (б) при $\mathrm{Re}_{1}=4,6 \times 10^{6} \mathrm{M}^{-1}$ Fig. 5. Curve of growth of disturbances $(a)$ at $\mathrm{z}=60 \mathrm{~mm}$ and evolution of amplitude-frequency spectra $(b)$ at $\operatorname{Re}_{1}=4.6 \times 10^{6} \mathrm{~m}^{-1}$

Кривая нарастания возмущений и полученные по этим данным амплитудно-частотные спектры при $\mathrm{M}=2$ и $\mathrm{Re}_{1}=4,6 \times 10^{6} \mathrm{M}^{-1}$ представлены на рис. 5. В работе [9] было выполнено сравнение экспериментальных степеней нарастания с результатами расчетов для модели крыла с углом скольжения передней кромки $\chi=45^{\circ}$ при $\mathrm{M}=2$ и $\mathrm{Re}_{1}=5 \times 10^{6} \mathrm{M}^{-1}$ и $x=80 \mathrm{мм}$. Получено хорошее согласование экспериментальных и теоретических данных в области линейного развития возмущений $\left(\operatorname{Re}_{x} \leq 0,6 \times 10^{6}\right)$. Данные, представленные на рис. 5 будут использоваться для сравнения скорости пространственного нарастания естественных возмущений, полученных путем обработки экспериментальных спектров с результатами расчета по линейной теории устойчивости.

\section{Заключение}

Проведены исследования влияния единичного числа Рейнольдса на ламинарно-турбулентный переход в сверхзвуковом пограничном слое скользящего крыла с дозвуковой передней кромкой. Показано, что ламинарно-турбулентный переход в сверхзвуковом пограничном слое скользящего крыла с дозвуковой передней кромкой наступает раньше $(\approx 25 \div 30 \%)$, чем на модели со сверхзвуковой передней кромкой при одних и тех же параметрах набегающего потока. Увеличение единичного числа Рейнольдса $\operatorname{Re}_{1}$ приводит к незначительному росту числа Рейнольдса перехода $\mathrm{Re}_{\mathrm{tr}}$. Изменение единичного числа Рейнольдса слабо влияет на ламинарно-турбулентный переход в пограничном слое скользящего крыла с дозвуковой передней кромкой, в отличие от модели крыла со сверхзвуковой передней кромкой. Причиной такого влияния может быть определяющая роль стационарных возмущений в процессе ламинарно-турбулентного перехода на модели скользящего крыла с дозвуковой передней кромкой.

\section{Список литературы}

1. Ермолаев Ю. Г., Косинов А. Д., Семенов А. Н., Семенов Н. В., Яцких А. А. Влияние единичного числа Рейнольдса на ламинарно-турбулентный переход на скользящем крыле при сверхзвуковых скоростях потока // Теплофизика и аэромеханика. 2018. Т. 25, № 5. C. 685-692. DOI 10.1134/S08698643180500 
2. Chapman G. T. Transition of the laminar boundary layer on a delta wing with 74 deg. sweep in free flights at Mach numbers from 2.8 to 5.3. In: NASA TN D-1066. 1961.

3. Cattafesta L. N. III, Iyer V., Masad J. A., King R. A., Dagenhart J. R. Three-dimensional boundary-layer transition on a swept wing at Mach 3.5. AIAA J., 1995, vol. 33, no. 11, p. 2032-2037.

4. Sugiura H., Yoshida K., Tokugawa N., Takagi S., Nishizawa A. Transition measurements on the natural laminar flow wing at Mach 2. J. of Aircraft, 2002, vol. 39, no. 6, p. 996-1002.

5. Kwak D-Y., Tokugawa N., Yoshida K. Validation of aerodynamic design technology of supersonic experimental airplane (nexst-1) by flight test. In: West-East High Speed Flow Field Conference. Moscow, 2007, p. 1-13.

6. Shi M., Zhu W., Lee C. Crossflow instability of hypersonic flow over an elliptic cone. In: AIAA Aviation 2020 forum. June 15-19, 2020 (virtual event). DOI 10.2514/6.2020-3073

7. Kosinov A. D., Semionov N. V., Yermolaev Yu. G. Disturbances in test section of T-325 supersonic wind tunnel. Novosibirsk, 1999, 24 p. (Preprint Institute of Theoretical and Applied Mechanics, № 6-99).

8. Semionov N. V., Yermolaev Yu. G., Kosinov A. D., Kocharin V. L., Panina A. V., Semenov A. N., Shipul S. A., Yatskikh A. A. Experimental study of the laminar-turbulent transition on models of wings with subsonic and supersonic leading edge at $\mathrm{M}=2$. Journal of Physics: Conf. Series, 2019, vol. 1404, p. 012097(5). DOI 10.1088/1742-6596/1404/1/012097

9. Semionov N. V., Yermolaev Yu. G., Kocharin V. L., Kosinov A. D., Semenov A. N., Smorodsky B. V., Yatskikh A. A. An effect of small angle of attack on disturbances evolution in swept wing boundary layer at Mach number $\mathrm{M}=2$. AIP Conf. Proc., 2018, vol. 2027, no. 1 , p. 030156(6). DOI 10.1063/1.5065250.

\section{References}

1. Ermolaev Y. G., Kosinov A. D., Semenov A. N., Semionov N. V., Yatskikh A. A. Effect of unit Reynolds number on the laminar-turbulent transition on a swept wing in supersonic flow. Thermophysics and Aeromechanics, 2018, vol. 25, no. 5, p. 659-665.

2. Chapman G. T. Transition of the laminar boundary layer on a delta wing with 74 deg. sweep in free flights at Mach numbers from 2.8 to 5.3. In: NASA TN D-1066. 1961.

3. Cattafesta L. N. III, Iyer V., Masad J. A., King R. A., Dagenhart J. R. Three-dimensional boundary-layer transition on a swept wing at Mach 3.5. AIAA J., 1995, vol. 33, no. 11, p. 2032-2037.

4. Sugiura H., Yoshida K., Tokugawa N., Takagi S., Nishizawa A. Transition measurements on the natural laminar flow wing at Mach 2. J. of Aircraft, 2002, vol. 39, no. 6, p. 996-1002.

5. Kwak D-Y., Tokugawa N., Yoshida K. Validation of aerodynamic design technology of supersonic experimental airplane (nexst-1) by flight test. In: West-East High Speed Flow Field Conference. Moscow, 2007, p. 1-13.

6. Shi M., Zhu W., Lee C. Crossflow instability of hypersonic flow over an elliptic cone. In: AIAA Aviation 2020 forum. June 15-19, 2020 (virtual event). DOI 10.2514/6.2020-3073

7. Kosinov A. D., Semionov N. V., Yermolaev Yu. G. Disturbances in test section of T-325 supersonic wind tunnel. Novosibirsk, 1999, 24 p. (Preprint Institute of Theoretical and Applied Mechanics, № 6-99).

8. Semionov N. V., Yermolaev Yu. G., Kosinov A. D., Kocharin V. L., Panina A. V., Semenov A. N., Shipul S. A., Yatskikh A. A. Experimental study of the laminar-turbulent transition on models of wings with subsonic and supersonic leading edge at $\mathrm{M}=2$. Journal of Physics: Conf. Series, 2019, vol. 1404, p. 012097(5). DOI 10.1088/1742-6596/1404/1/012097

9. Semionov N. V., Yermolaev Yu. G., Kocharin V. L., Kosinov A. D., Semenov A. N., Smorodsky B. V., Yatskikh A. A. An effect of small angle of attack on disturbances evolution in 
swept wing boundary layer at Mach number $\mathrm{M}=2$. AIP Conf. Proc., 2018, vol. 2027, no. 1, p. 030156(6). DOI 10.1063/1.5065250.

Материал поступил в редколлегию

\section{Сведения об авторах / Information about the Authors}

Кочарин Василий Леонидович, младший научный сотрудник, Институт теоретической и прикладной механики им С. А. Христиановича СО РАН (Новосибирск, Россия)

Vasilii L. Kocharin, Junior Researcher, Khristianovich Institute of Theoretical and Applied Mechanics SB RAS (Novosibirsk, Russian Federation)

kocharin@itam.nsc.ru

Семёнов Николай Васильевич, доктор физико-математических наук, старший научный сотрудник, ведущий научный сотрудник, Институт теоретической и прикладной механики им С. А. Христиановича СО РАН (Новосибирск, Россия)

Nikolai V. Semionov, Doctor of Science (Physics and Mathematics), Senior Researcher, Leading Researcher, Khristianovich Institute of Theoretical and Applied Mechanics SB RAS (Novosibirsk, Russian Federation)

semion@itam.nsc.ru

Косинов Александр Дмитриевич, доктор физико-математических наук, старший научный сотрудник, заведующий лабораторией, Институт теоретической и прикладной механики им С. А. Христиановича СО РАН (Новосибирск, Россия)

Alexander D. Kosinov, Doctor of Science (Physics and Mathematics), Senior Researcher, Head of Laboratory, Khristianovich Institute of Theoretical and Applied Mechanics SB RAS (Novosibirsk, Russian Federation)

kosinov@itam.nsc.ru

Яцких Алексей Анатольевич, кандидат физико-математических наук, младший научный сотрудник, Институт теоретической и прикладной механики им С. А. Христиановича СО РАН (Новосибирск, Россия); младший научный сотрудник, Новосибирский государственный университет (Новосибирск, Россия)

Aleksey A. Yatskikh, PhD, Junior Researcher, Khristianovich Institute of Theoretical and Applied Mechanics SB RAS (Novosibirsk, Russian Federation); Junior Researcher, Novosibirsk State University (Novosibirsk, Russian Federation)

73.yatskikh@gmail.com 
Шипуль Софья Андреевна, старший лаборант с высшим профессиональным образованием, Институт теоретической и прикладной механики им С. А. Христиановича СО РАН (Новосибирск, Россия)

Sofia A. Shipul, PhD Student, Khristianovich Institute of Theoretical and Applied Mechanics SB RAS (Novosibirsk, Russian Federation)

sofa.shipul@gmail.com

Ермолаев Юрий Геннадьевич, кандидат физико-математических наук, старший научный сотрудник, Институт теоретической и прикладной механики им С. А. Христиановича СО РАН (Новосибирск, Россия)

Yury G. Yermolaev, PhD, Senior Fellow, Khristianovich Institute of Theoretical and Applied Mechanics SB RAS (Novosibirsk, Russian Federation)

yermol@itam.nsc.ru 